\title{
An assessment of serial co-cultivation approach for generating novel Zymomonas mobilis strains
}

\author{
Katsuya Fuchino* ${ }^{*}$ and Per Bruheim
}

\begin{abstract}
Objective: The alphaproteobacterium Zymomonas mobilis is an efficient ethanol producer, and Z. mobilis-based biorefinery shows great potential for biofuel production. Serial co-cultivation is an emerging approach that promotes inter-species interactions which can improve or rewire the metabolic features in industrially useful microorganisms by inducing frequent mutations. We applied this method to assess if it improves or rewires the desirable physiological features of Z. mobilis, especially ethanol production.

Results: We performed serial co-culture of Z. mobilis with the baker's yeast, Saccharomyces cerevisiae. We observed filamentation of $Z$. mobilis cells in the co-culture, indicating that the $Z$. mobilis cells were exposed to stress due to the presence of a competitor. After 50 times of serial transfers, we characterized the generated Z. mobilis strains, showing that long term co-culture did not drive significant changes in either the growth or profile of excreted metabolites in the generated strains. In line with this, whole genome sequencing of the generated $Z$. mobilis strains revealed only minor genetic variations from the parental strain. 50 generations of $Z$. mobilis monoculture did not induce morphological changes or any significant genetic variations. The result indicates that the method needs to be carefully optimized for Z. mobilis strain improvement.
\end{abstract}

Keywords: Zymomonas mobilis, Ethanol production, Adaptive evolution, Co-culture, Genomic stability

\section{Introduction}

The alphaproteobacterium $Z$. mobilis is the best bacterial ethanol producer endowed with unique physiological features [1]. Therefore, Z. mobilis based-biorefinery is a promising biofuel production system at an industrial scale [2]. Despite its efficient ethanol production capacity, there are challenges in employing $Z$. mobilis as a biocatalyst. For example, $Z$. mobilis consumes a limited range of feedstock as substrate [2]. It is also known to be sensitive to certain abiotic stress, such as acetic acid toxicity $[2,3]$. Yet, recent advances in $Z$. mobilis metabolic engineering

*Correspondence: katsuyafuchino@gmail.com

Department of Biotechnology and Food Science, Norwegian University of Science and Technology, Trondheim, Norway have overcome these drawbacks, advancing its potential use for environmentally-friendly biorefining [2, 3].

In addition to ethanol production, rewiring metabolic pathways to produce other useful compounds has lately been explored in Z. mobilis [3-7]. This is to utilize and exploit its intrinsic capacity of fast catabolism that comes with small biomass accumulation [1]. Considering that its prolific potential is expanding, a novel approach to engineer or generate desirable $Z$. mobilis strains should be assessed.

Co-culture based adaptive evolution involves mixing several species in the same culture to stimulate interspecies interactions, this novel and recent approach improves the physiological features of industrially beneficial microorganisms by inducing frequent mutations [8]. For example, serial co-culture of Candida glabrata and 
Pichia kudriavzevii significantly influenced the growth and fermentation profile of co-evolved strains [9]. The evolved strains conferred altered chemical complexity in produced wine [9]. Zhou et al. showed that long term bacterial-yeast competition induced chromosomal arrangements in the yeast, rendering stress-tolerance, altered metabolism and other physiological features in the yeast Lachancea kluyveri [10]. In addition to promoting mutation, co-culture has also been shown to promote the production of particular metabolites in Streptomyces species, which were otherwise not expressed in pure monoculture [11, 12]. Thus, inter-species interaction stimulates expression of cryptic genes. [11-13]. Such a response might also bias mutational events if the cryptic genes were continuously expressed during serial co-culture.

In addition to gaining potentially desirable traits, another advantage of the serial co-culture based adaptation method is to shed light on the understanding of basic ecological aspects of species interaction [14, 15]. Given that Z. mobilis ecology and its natural habitat is yet rather enigmatic [16], the approach should be worth being examined in this regard as well.

In the present work, we adopted serial co-culture of $Z$. mobilis mixed with baker's yeast Saccharomyces cerevisiae for strain generation. The aim of study was to see if $Z$. mobilis changes or rewires its ethanologenic features through competition and interaction with S. cerevisiae through serial co-cultivation.

\section{Main text \\ Method \\ Cultivation}

Z. mobilis Zm6 and S. cerevisiae strain CEN.PK113-7D strain were used for adaptive evolution by co-culture. Cells were grown in growth medium containing glucose $(20 \mathrm{~g} / \mathrm{L}$ or $100 \mathrm{~g} / \mathrm{L})$, yeast extract $(5 \mathrm{~g} / \mathrm{L}), \mathrm{NH}_{4} \mathrm{SO}_{4}$ $(1 \mathrm{~g} / \mathrm{L}), \mathrm{KH}_{2} \mathrm{PO}_{4}(1 \mathrm{~g} / \mathrm{L})$ and $\mathrm{MgSO}_{4}(0.5 \mathrm{~g} / \mathrm{L})$. The growth medium was flushed with nitrogen gas prior to culture. The co-culture was grown at $30^{\circ} \mathrm{C}$ in a tightly capped test tube with shaking at $200 \mathrm{rpm}$ throughout the study.

For serial co-culture, $10 \mu \mathrm{L}$ of $\mathrm{Zm} 6$ overnight anaerobic culture and S. cerevisiae anaerobic culture were used as starters. The mixed cultures typically spent all nutrients within a day. The fully grown cultures $(10 \mu \mathrm{L})$ were transferred to fresh identical medium the following day and the co-culture continued. After the transfer was repeated 5 times, we observed that $Z$. mobilis competes out in the co-culture under the condition, as shown by viable counts of the co-culture. To maintain balanced coculture, we inoculated additional $S$. cerevisiae overnight culture $40 \mu \mathrm{L}$, in addition to $10 \mu \mathrm{L}$ of previous co-culture, upon each transfer from 6th round.
For Escherichia coli co-culture with Z. mobilis, E. coli strain K12 grown in LB medium under aerobic condition was used as a starter. We started co-culture with mixing $10 \mu \mathrm{L}$ of fully grown monocultures of $Z$. mobilis and E. coli. During the experiments, we learned that inoculating $10 \mu \mathrm{L}$ of fully grown co-culture of previous round with $20 \mu \mathrm{L}$ of fully grown $E$. coli monoculture upon a transfer gave a good balance for continuation of co-cultures, which we performed for all transfer. All lines were replicated for whole passages.

After the serial co-culture of 50 transfers, cells were streaked out on the solid identical medium. Isolated pure strains are designated as follows, Zs100: Z. mobilis $\mathrm{Zm} 6$ derived strain obtained from last round of $Z$. mobilis vs $S$. cerevisiae serial co-culture supplemented with glucose $100 \mathrm{~g} / \mathrm{L}$. Zs100R is obtained from a parallel replicate of $Z$. mobilis vs $S$. cerevisiae glucose $100 \mathrm{~g} / \mathrm{L}$. Zs20: Zm6 derived strain obtained from the last round of serial co-culture with $S$. cerevisiae supplemented with $20 \mathrm{~g} / \mathrm{L}$ glucose, and Zs20R was obtained from a last culture of parallel run of Zs20. Similarly, Ze20 designates $Z$. mobilis strain obtained from last round of $Z$. mobilis vs $E$. coli co-culture with $20 \mathrm{~g} / \mathrm{L}$ glucose, and Ze20R as a parallel replicate. As a control, the 50 times serial transfer of $Z$. mobilis monocultures grown in the same complex medium with $20 \mathrm{~g} / \mathrm{L}$ glucose or $100 \mathrm{~g} / \mathrm{L}$ glucose were performed. Z20 designates $Z$. mobilis strain obtained from a last round of $Z$. mobilis monoculture with $20 \mathrm{~g} / \mathrm{L}$ glucose, and Z100 designates $Z$. mobilis strain obtained from a last round of monoculture with $100 \mathrm{~g} / \mathrm{L}$ glucose.

\section{Characterization of growth and ethanol production}

Growth profiles of all strains were analyzed using flat bottom 96-well microplate in plate-reader spark $20 \mathrm{M}$ (Tecan) by measuring its absorbance at $600 \mathrm{~nm}$. Overnight anaerobic monoculture was used as an inoculum. Temperature control was set at $30{ }^{\circ} \mathrm{C}$. Three technical replicates were repeated for each time point. The used medium in plate reader was same as for co-culture, with supplement of glucose $20 \mathrm{~g} / \mathrm{L}$. We also measured growth profiles in test tubes under anaerobic condition using spectrophotometer (VWR), showing similar trends from microplate experiments.

Acetate, lactate, ethanol and glucose in the overnight monoculture of generated $Z$. mobilis strains were measured using Waters 2695e Alliance HPLC (Waters) with Hi-plex column $(300 \times 7.7 \mathrm{~mm}$, Agilent). The spent medium of anaerobic overnight culture in the test tube was analyzed for HPLC analysis. The collected supernatant was filtered through $0.2 \mu \mathrm{m}$ Supor $^{\circledR}$ polyether sulfone membrane (PALL) before the analysis. HPLC 
analysis was run under the condition; $0.05 \mathrm{M}$ sulfuric acid as mobile phase at a flow rate of $0.8 \mathrm{~mL} /$ minute. External standard curve was used for converting obtained peaks to concentration of analytes.

\section{Microscopy}

Growing sample was directly mounted on Phosphatebuffered saline (PBS)-agarose pad before imaging. Zeiss Axio Imager Z2 microscope (ZEISS) equipped with camera Axiocam MR R3 (ZEISS) was used for capturing phase contrast images. Software ZEN 3.1 (ZEISS) was used for image analysis.

\section{Whole genome sequencing}

Total DNA was extracted by combining a lysozyme treatment [17] and D-neasy blood tissue kit (Qiagen). Whole genome of the co-cultured $Z$. mobilis strains was sequenced by GATC re-sequencing service (INVIEW Genome sequencing). The reference sequence was our lab stock Zm6 strain which was previously sequenced $[18,19]$.

\section{Results}

In order to generate novel $Z$. mobilis strains, we assessed the co-culturing based adaptive evolution. We chose serial transfer instead of continuous chemostat co-culture, for the simplicity of method, and baker's yeast $S$. cerevisiae as a competitor since it also produces ethanol and likely share an ecological niche with $Z$. mobilis, although ethanol production pathway differs between two species. During the pilot experiment, we observed that many Z. mobilis cells showed abnormal cell shape in the co-culture with $S$. cerevisiae supplemented with 100 g/L glucose (Fig. 1). Most of Z. mobilis cells formed filamentous structures which can be up to 10 times longer than regular cell (Fig. 1, shown as $C$ in the lowerright panel). Some of the $Z$. mobilis cells were burst or exhibiting a membrane protrusion from cell (Fig. 1, shown as A in lower-right panel) and several cells were found dead as shown by lighter phase contrast (Fig. 1b). Dead S. cerevisiae cells were also observed (Fig. 1, shown as D in lower-right panel). It should be noted that these morphological changes or death of $Z$. mobilis cells were not observed in the $Z$. mobilis monoculture using the same growth condition. Bacterial filamentation is often an adaption to stress [20]. Thus, we found the observed phenomenon as a good indication that the Zm6 cells were interacting and competing with S. cerevisiae. This prompted us to expect that frequent mutations should arise in the $Z$. mobilis cells to gain advantages for the competition during serial co-culture. In the co-culture
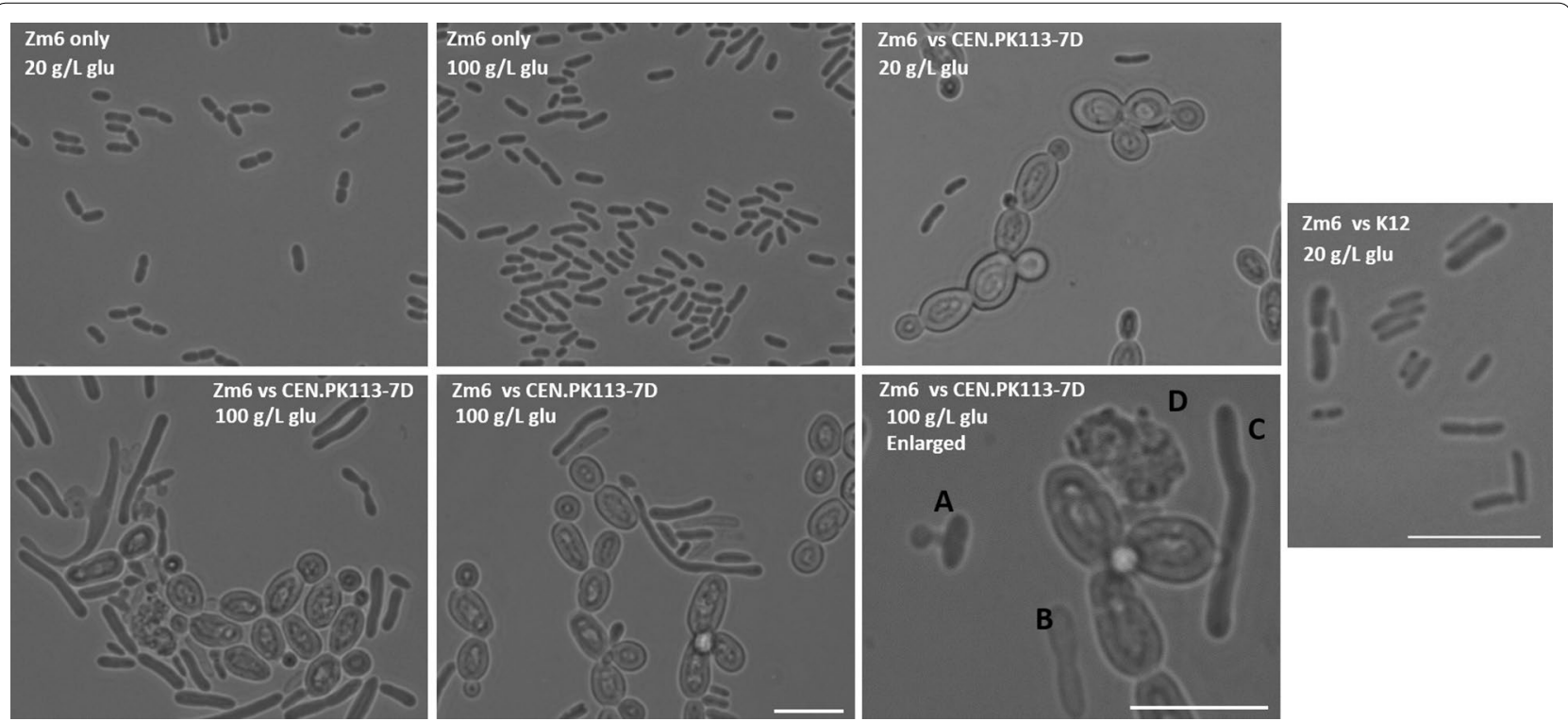

Fig. 1 Phase contrast images of Z. mobilis monoculture and co-culture with S. cerevisiae strain CEN.PK113-7D or E. coli strain K12. Used strains and conditions are as shown in white texts in the images. Abbreviation glu stands for glucose in the medium. It is to be noted that $Z$. mobilis strain in the co-culture vs yeast with $100 \mathrm{~g} / \mathrm{L}$ glucose formed elongated filamentous structure, while other conditions did not induce such drastic morphological changes in Z. mobilis cells. Right-bottom panel and Zm6 vs K12 panel are enlarged images with scale bar $10 \mu \mathrm{m}$, while all other panel sizes are corresponding with the scale bar $10 \mu \mathrm{m}$, found in the bottom-middle panel. Black $\mathbf{a}$ in the right-bottom panel indicates bursting cell with membrane protrusion from the cytoplasm. b The lysed cell exhibiting lighter phase contrast than that of the live cells. $\mathbf{c}$ Elongated Z. mobilis cell. d Dead yeast cell 
with E. coli, obvious morphological changes of Z. mobilis and $E$. coli was not observed.

We then performed serial transfer of each mixed culture for 50 passages. For co-culture with S. cerevisiae, we made two lines of culture supplemented with $20 \mathrm{~g} / \mathrm{L}$ or $100 \mathrm{~g} / \mathrm{L}$ glucose. For E. coli co-culture, we performed cultivation only with $20 \mathrm{~g} / \mathrm{L}$ glucose. All co-cultures were run with parallel replicates, resulting in 6 lines of serial co-cultures. In addition, we performed serial transfer of two monocultures of $Z$. mobilis strains for 50 passages as controls.

After 50 transfers of serial co-cultures, we characterized growth profile and ethanol production by monoculture of obtained $Z$. mobilis strains from last batch of long-term co-cultures. It was to be noted that the elongated morphology observed during serial co-culture was not retained in $Z$. mobilis cells grown in monoculture, suggesting that filamentation was due to stress. The HPLC and growth curve data showed that excreted production and growth profiles by obtained strains were nearly identical to those by parental strain (Fig. 2). This was somewhat contrary to what we anticipated, especially in the $Z$. mobilis cells with $S$. cerevisiae in $100 \mathrm{~g} / \mathrm{L}$ glucose, since the $Z$. mobilis cells in that condition underwent long cultivation under constant stress, which could have influenced its metabolism or growth profile for better survival. This result is in sharp contrast to our previous experience with $Z$. mobilis adaptive evolution against salt stress, in which 10-20 passages of serial transfer drove the strains to gain adapted phenotype of two times better total ethanol production under the saline condition than that of the parental strain [19]. The obtained result here showed that the competition with yeast or $E$. coli in the co-culture did not influence $Z$. mobilis ethanologenic physiology.

Next, to see if there are mutations not reflected in the measured phenotypes, we sequenced whole genome of the obtained $Z$. mobilis strains. The analysis revealed that several mutations in the ORF regions of the generated strains, compared to the reference genome [18]. Yet, it was not clear how the mutations could relate to advantage in the species interaction. Interestingly, Z. mobilis strain Zs100R did not show any mutation in the ORF regions, despite long-term constant competition with $S$. cerevisiae. The strain Z20 obtained from serial-monoculture did not show any mutations either. This suggests that genomic stability was very high in $Z$. mobilis during the serial co-culture and monoculture.

\section{Discussion}

Even though co-culture based adaptive evolution has shown to be a powerful tool for several bacterial and
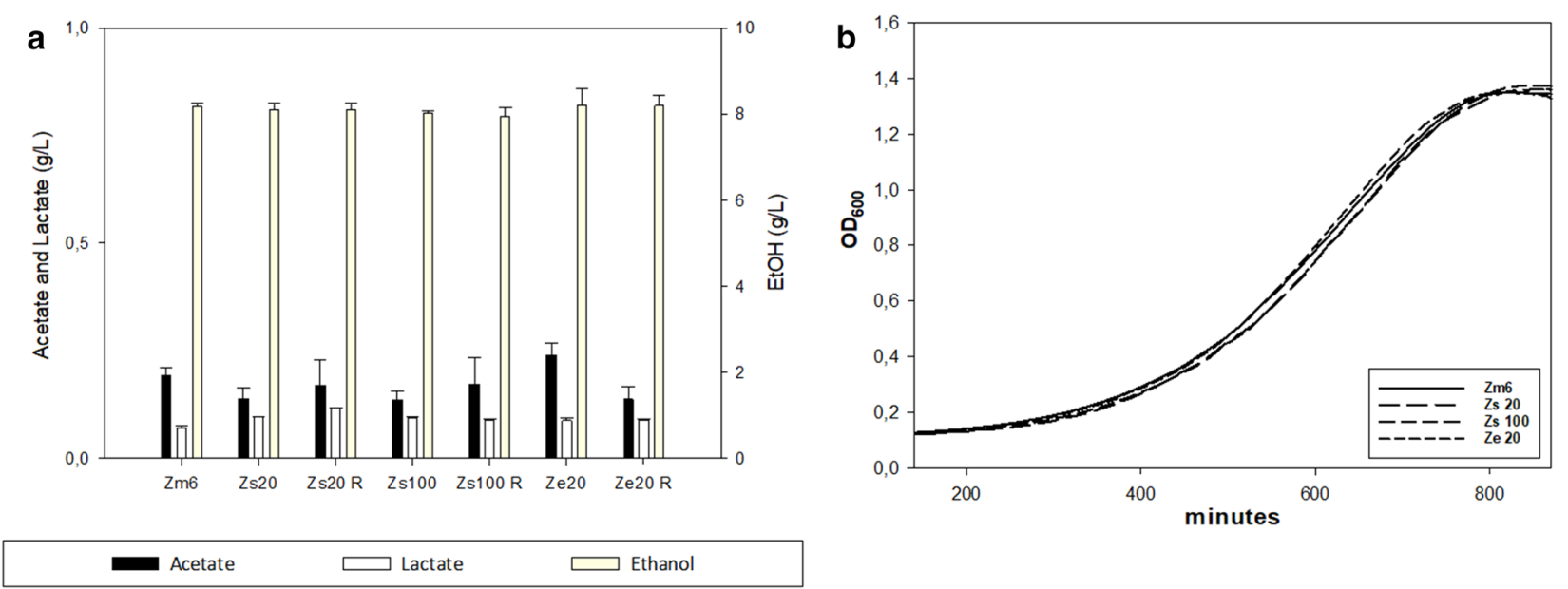

Fig. 2 Characterization of $Z$. mobilis strains that generated from serial co-culture-based laboratory adaptation. a Total production of acetate, lactate and ethanol from the overnight monoculture of the generated Z. mobilis strains and the parental strain Zm6. $\mathbf{b}$ Growth profiles of monoculture $Z$. mobilis strain obtained in this study and the parental strain Zm6 in the complex medium with glucose $20 \mathrm{~g} / \mathrm{L}$. Z. mobilis strain designations are as follows; Zs100; Z. mobilis strain obtained from the last round of Z. mobilis vs S. cerevisiae serial co-culture with glucose $100 \mathrm{~g} / \mathrm{L}$, Zs100R; obtained from a parallel replicate of Z. mobilis vs S. cerevisiae glucose $100 \mathrm{~g} / \mathrm{L}, \mathrm{Zs} 20 ; Z$. mobilis strain obtained from the last round of serial co-culture with $S$. cerevisiae supplemented with $20 \mathrm{~g} / \mathrm{L}$ glucose, Zs20R; Z. mobilis strain from a last culture of parallel run of Zs20, Ze20; Z. mobilis strain obtained from last round of Z. mobilis vs E. coli co-culture with $20 \mathrm{~g} / \mathrm{L}$ glucose, and Ze20R as a parallel replicate of Ze20, Z20 designates Z. mobilis strain obtained from a last round of $Z$. mobilis monoculture with $20 \mathrm{~g} / \mathrm{L}$ glucose, and Z100 designates $Z$. mobilis strain obtained from a last round of monoculture with $100 \mathrm{~g} / \mathrm{L}$ glucose. Generation time for actively growing Z. mobilis strains in the plate reader was as follows; $175 \mathrm{~min}$ for Zm6, $177 \mathrm{~min}$ for Zs 20 , 173 min for Zs100 and 179 min for Ze20. Note that there were no significant differences in ethanol production and growth profiles between strains. Error bars; standard deviations from 3 independent measurements 
Table 1 A table of list of mutations found in generated Z. mobilis strains. Note that all the strains were obtained from the last round of serial co-culture or monoculture. NA stands for not available

\begin{tabular}{|c|c|c|c|c|c|c|}
\hline Strain & Serial co-culture method & $\begin{array}{l}\text { Position } \\
\text { of the genome }\end{array}$ & Referencee & Mutation & Gene & Annotation \\
\hline Zs100 & $\begin{array}{l}\text { Z. mobilis vs S. cerevisiae in the medium } \\
\text { with } 100 \mathrm{~g} / \mathrm{L} \text { glucose }\end{array}$ & 87016 & GA & G & ZZ6_0076 & Hypothetical protein \\
\hline Zs100R & $\begin{array}{l}\text { Z. mobilis vs S. cerevisiae in the medium } \\
\text { with } 100 \mathrm{~g} / \mathrm{L} \text { glucose, replicate }\end{array}$ & NA & & NA & & \\
\hline Zs20 & $\begin{array}{l}\text { Z. mobilis vs S. cerevisiae in the medium } \\
\text { with } 20 \mathrm{~g} / \mathrm{L} \text { glucose }\end{array}$ & NA & & NA & & \\
\hline Zs20R & $\begin{array}{l}\text { Z. mobilis vs S. cerevisiae in the medium } \\
\text { with } 20 \mathrm{~g} / \mathrm{L} \text { glucose, replicate }\end{array}$ & 114095 & C & $\mathrm{T}$ & ZZ6_0103 & Hypothetical protein \\
\hline Ze20 & $\begin{array}{l}\text { Z. mobilis vs E. coli in the medium with } \\
20 \mathrm{~g} / \mathrm{L} \text { glucose }\end{array}$ & 1195056 & C & $\mathrm{T}$ & ZZ6_1040 & TonB-dependent receptor \\
\hline Ze20R & $\begin{array}{l}\text { Z. mobilis vs } E \text {. coli in the medium with } \\
20 \mathrm{~g} / \mathrm{L} \text { glucose, replicate }\end{array}$ & 1185646 & A & AGGCTCAG & ZZ6_1032 & $\begin{array}{l}\text { Heptose-I-phosphate ethanola- } \\
\text { minephosphotransferase }\end{array}$ \\
\hline Z20 & $\begin{array}{l}\text { Z. mobilis monoculture in the medium } \\
\text { with } 20 \mathrm{~g} / \mathrm{L} \text { glucose }\end{array}$ & NA & & NA & & \\
\hline Z100 & $\begin{array}{l}\text { Z. mobilis monoculture in the medium } \\
\text { with } 100 \mathrm{~g} / \mathrm{L} \text { glucose }\end{array}$ & 531639 & G & A & ZZ6_4600 & UDP-N-acetylmuramate-L-alanine ligase \\
\hline
\end{tabular}

yeast species, we recommend that the method should be carefully fine-tuned for $Z$. mobilis. Our data suggest that serial co-culture methods utilizing speciesinteraction did not drive mutations in the genome of $Z$. mobilis strains in our experimental setting. Although the presence of $S$. cerevisiae induced stressed shape of $Z$. mobilis, the genome content of $Z$. mobilis cells was found almost intact (Table 1). Perhaps, utilizing more 'stressful' organism to $Z$. mobilis in the culture might drive the need of better fitness in $Z$. mobilis and thus improve an outcome of the method. Alternatively, $Z$. mobilis genomic stability might be very stable during species interaction. Z. mobilis was previously suggested to possess polyploidy [21, 22], and that could render genetic stability during stress responses by frequent homologous recombination. Confirmation of polyploidy and identification of copy numbers of chromosome in Z. mobilis cells should be useful knowledge for $Z$. mobilis research. Interestingly, recent report suggests that $Z$. mobilis is not involved in pair-wise cross feeding with other bacteria [23], implying that $Z$. mobilis does not cooperate with other microbes for its survival.

\section{Limitation}

Apart from growth curves and excreted metabolites profiles, there might be hidden phenotypes in generated strains that we did not detect. Changes in the genome of $S$. cerevisiae after the serial co-culture with Z. mobilis was not followed in this study. An investigation on
S. cerevisiae strains that are co-cultured for long terms with $Z$. mobilis under appropriate experimental settings might be a future line of investigation.

\section{Abbreviations}

HPLC: High-pressure liquid chromatography; OD: Optical Density; ORF: Open Reading Frame.

\section{Acknowledgements}

The authors thank Dr. Kåre Andre Kristiansen and Ida Eide Langørgen for their technical assistances. The authors are grateful to Stuart Heggs for his critical reading on the manuscript.

\section{Authors' contributions}

KF conceptualized and designed the study, conducted the experiments, interpreted and analyzed the data, wrote and edited the draft manuscript. PB supervised the study and experiments, interpreted and analyzed the data, edited the draft manuscript. All authors read and approved the final manuscript.

\section{Funding}

The current work is supported by the Research Council of Norway, grant number 258657

\section{Availability of data and materials}

The datasets, sequenced genomes of the studied strains, and strains generated from the present work are available from the corresponding author upon on a reasonable request.

Ethics approval and consent to participate Not applicable.

Consent for publication

Not applicable.

\section{Competing interests}

The authors declare that they have no competing interests. 
Received: 20 May 2020 Accepted: 28 August 2020

Published online: 07 September 2020

\section{References}

1. Kalnenieks U: Physiology of Zymomonas mobilis: Some Unanswered Questions. In: Advances in Microbial Physiology. Edited by Poole RK, vol. 51: Academic Press; 2006: 73-117.

2. He MX, Wu B, Qin H, Ruan ZY, Tan FR, Wang JL, Shui ZX, Dai LC, Zhu QL, Pan K, et al. Zymomonas mobilis: a novel platform for future biorefineries. Biotechnol Biofuels. 2014;7(1):101.

3. Wang $X$, He Q, Yang Y, Wang J, Haning K, Hu Y, Wu B, He M, Zhang Y, Bao J, et al. Advances and prospects in metabolic engineering of Zymomonas mobilis. Metab Eng. 2018;50:57-73.

4. Yang S, Mohagheghi A, Franden MA, Chou Y-C, Chen X, Dowe N, Himmel ME, Zhang M. Metabolic engineering of Zymomonas mobilis for 2,3-butanediol production from lignocellulosic biomass sugars. Biotechnol Biofuels. 2016;9(1):189.

5. Qiu M, Shen W, Yan X, He Q, Cai D, Chen S, Wei H, Knoshaug EP, Zhang $M$, Himmel ME, et al. Metabolic engineering of Zymomonas mobilis for anaerobic isobutanol production. Biotechnol Biofuels. 2020;13(1):15

6. Kalnenieks U, Balodite E, Strähler S, Strazdina I, Rex J, Pentjuss A, Fuchino K, Bruheim P, Rutkis R, Pappas KM et al: Improvement of Acetaldehyde Production in Zymomonas mobilis by Engineering of Its Aerobic Metabolism. Front Microbio 2019, 10(2533).

7. Yang S, Fei Q, Zhang Y, Contreras LM, Utturkar SM, Brown SD, Himmel ME, Zhang M. Zymomonas mobilis as a model system for production of biofuels and biochemicals. Microb Biotechnol. 2016;9(6):699-717.

8. Swamy KBS, Zhou N. Experimental evolution: its principles and applications in developing stress-tolerant yeasts. Appl Microbiol Biotechnol. 2019;103(5):2067-77.

9. Morrison-Whittle P, Lee SA, Fedrizzi B, Goddard MR: Co-evolution as Too for Diversifying Flavor and Aroma Profiles of Wines. Frontiers Microbiol 2018, 9(910).

10. Zhou N, Bottagisi S, Katz M, Schacherer J, Friedrich A, Gojkovic Z, Swamy KBS, Knecht W, Compagno C, Piškur J: Yeast-bacteria competition induced new metabolic traits through large-scale genomic rearrangements in Lachancea kluyveri. FEMS Yeast Research 2017, 17(6).

11. Pishchany G, Mevers E, Ndousse-Fetter S, Horvath DJ, Paludo CR, SilvaJunior EA, Koren S, Skaar EP, Clardy J, Kolter R. Amycomicin is a potent and specific antibiotic discovered with a targeted interaction screen. Proc Natl Acad Sci. 2018;115(40):10124.
12. Traxler MF, Kolter R. Natural products in soil microbe interactions and evolution. Nat Product Rep. 2015;32(7):956-70.

13. Okada BK, Seyedsayamdost MR. Antibiotic dialogues: induction of silent biosynthetic gene clusters by exogenous small molecules. FEMS Microbiol Rev. 2017;41(1):19-33.

14. Conacher CG, Rossouw D, Bauer FFB: Peer pressure: evolutionary responses to biotic pressures in wine yeasts. FEMS Yeast Research 2019, 19(7).

15. Lenski RE. Experimental evolution and the dynamics of adaptation and genome evolution in microbial populations. ISME J. 2017;11(10):2181-94.

16. Weir PM. The ecology of Zymomonas: a review. Folia Microbiol. 2016;61(5):385-92.

17. Gill C, van de Wijgert JHHM, Blow F, Darby AC. Evaluation of Lysis Methods for the Extraction of Bacterial DNA for Analysis of the Vaginal Microbiota. PLoS ONE. 2016;11(9):e0163148.

18. Desiniotis A, Kouvelis VN, Davenport K, Bruce D, Detter C, Tapia R, Han C, Goodwin LA, Woyke T, Kyrpides NC et al: Complete Genome Sequence of the Ethanol-Producing Zymomonas mobilis subsp. mobilis Centrotype ATCC 29191. J Bacteriol. 2012, 194(21):5966.

19. Fuchino K, Bruheim P. Increased salt tolerance in Zymomonas mobilis strain generated by adaptative evolution. Microb Cell Fact. 2020;19(1):147

20. Rizzo MG, De Plano LM, Franco D: Regulation of filamentation by bacteria and its impact on the productivity of compounds in biotechnological processes. Applied Microbiology and Biotechnology 2020.

21. Skerker JM, Leon D, Price MN, Mar JS, Tarjan DR, Wetmore KM, Deutschbauer AM, Baumohl JK, Bauer S, Ibáñez AB, et al. Dissecting a complex chemical stress: chemogenomic profiling of plant hydrolysates. Mol Syst Biol. 2013;9(1):674.

22. Brenac L, Baidoo EEK, Keasling JD, Budin I. Distinct functional roles for hopanoid composition in the chemical tolerance of Zymomonas mobilis. Mol Microbiol. 2019;112(5):1564-75.

23. Pacheco AR, Moel M, Segrè D. Costless metabolic secretions as drivers of interspecies interactions in microbial ecosystems. Nature Communications. 2019;10(1):103.

\section{Publisher's Note}

Springer Nature remains neutral with regard to jurisdictional claims in published maps and institutional affiliations.
Ready to submit your research? Choose BMC and benefit from:

- fast, convenient online submission

- thorough peer review by experienced researchers in your field

- rapid publication on acceptance

- support for research data, including large and complex data types

- gold Open Access which fosters wider collaboration and increased citations

- maximum visibility for your research: over 100M website views per year

At BMC, research is always in progress.

Learn more biomedcentral.com/submissions 